\title{
On some special kind of space-times, II
}

Dedicated to Professor Yoshie Katsurada on her Sixtieth Birthday

\section{By Hyôitirô TAKeno and Shin-ichi Kitamura}

\section{§1. Introduction.}

The present paper is a continuation of the paper under the same title $[1]^{1)}$ and makes clear some geometric properties of the space-time $\mathrm{V}$ which will be of use when we deal with the problem of the freedom of the characteristic system. The same notations and terminologies as those in [1] will be used.

We shall first give some results in [1] which will play important roles in the present paper. $V$ is a 4-dimensional Riemannian space whose metric can be brought into the form

$$
d s^{2}=-d x^{2}-B d y^{2}-C d z^{2}+D d t^{2}, \quad\left(\left(x^{i}\right) \equiv(x, y, z, t)\right),
$$

where $B, C$ and $D$ are positive-valued functions of one variable $x$. A set of vectors $\stackrel{\alpha}{u}_{i},(\alpha, \beta, \cdots=1, \cdots, 4 ; i, j, \cdots=1, \cdots, 4)$, and scalars $\lambda_{a}, \mu_{a}, \lambda_{1 a}\left(=\lambda_{a 1}\right)$, $\lambda_{a b}\left(=\lambda_{b a}\right),(a, b=2,3,4)$, is defined in this coordinate system by

$$
\begin{aligned}
& \stackrel{1}{u_{i}}=\delta_{i}^{1}, \quad \stackrel{2}{u_{i}}=\sqrt{B} \delta_{i}^{2}, \quad \stackrel{3}{u_{i}}=\sqrt{C} \delta_{i}^{3}, \quad \stackrel{4}{u_{i}}=\sqrt{D} \delta_{i}^{4} ; \\
& \lambda_{2}=-\beta / 2, \quad \lambda_{3}=-\gamma / 2, \quad \lambda_{4}=-\delta / 2, \\
& \left(\beta \equiv B^{\prime} / B, \quad \gamma \equiv C^{\prime} / C, \quad \delta \equiv D^{\prime} / D ; ' \equiv d / d x\right) ; \\
& \mu_{2}=-\beta^{\prime} / 2, \quad \mu_{3}=-\gamma^{\prime} / 2, \quad \mu_{4}=-\delta^{\prime} / 2 ; \\
& \lambda_{1 a}=\left(\lambda_{a}\right)^{2}-\mu_{a}, \quad \lambda_{a b}=\lambda_{a} \lambda_{b}, \quad(a \neq b),
\end{aligned}
$$

The set is called a characteristic system (abbreviated to $c$.s.) of the $V$ under consideration.

These quantities satisfy the following tensor relations:

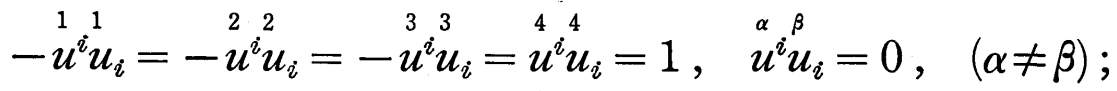

$$
\begin{aligned}
& \nabla_{i} \stackrel{1}{u_{j}}=-\lambda_{2} u_{i}^{2} u_{j}^{2}-\lambda_{3} u_{i}^{3} u_{j}+\lambda_{4}{ }_{4}^{4} u_{i}^{4} u_{j} \\
& \left.\nabla_{i}^{a} u_{j}=\lambda_{a}{ }_{a}^{a} u_{i} u_{j}, \quad \text { (not summed for } a\right) \text {; }
\end{aligned}
$$

1) Numbers in brackets refer to the references at the end of the paper. 


$$
\nabla_{i} \lambda_{a}=\mu_{a} u_{i}
$$

Further, we have

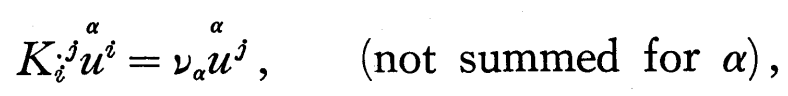

where $K_{i}^{j}$ is the Ricci tensor and $\nu^{\prime}$ s are the principal values (i. e. eigenvalues of the Ricci tensor), and

$$
\begin{array}{ll}
\nu_{1}=-\left(\lambda_{12}+\lambda_{13}+\lambda_{14}\right)\left(=K_{1}^{1}\right), & \nu_{2}=-\left(\lambda_{12}+\lambda_{23}+\lambda_{24}\right)\left(=K_{2}^{2}\right), \\
\nu_{3}=-\left(\lambda_{13}+\lambda_{23}+\lambda_{34}\right)\left(=K_{3}^{3}\right), & \nu_{4}=-\left(\lambda_{14}+\lambda_{24}+\lambda_{34}\right)\left(=K_{4}^{4}\right) .
\end{array}
$$

It was shown in [1] that a necessary and sufficient condition that a space-time be a $V$ is given by the existence of a set $\left\{\stackrel{\alpha}{u_{i}}, \lambda_{a}, \mu_{a}\right\}$ satisfying (1.6), (1.7) and (1.8). Further some relations satisfied by the members of c. s. are obtained and some fundamental theorems concerning the freedom of c.s. are proved. These results will be used without detailed explanations in the following.

As is stated at the beginning of this section, the purpose of the present paper is to make clear some invariant properties of $\mathrm{V}$, to classify V's using these investigations, and to make preparation for solving the problem of the freedom of c.s.

\section{§2. Classification of $V$ 's in terms of $\nu$ 's.}

As is seen from (1.9), $\stackrel{\alpha}{u_{i}}$ 's are eigenvectors of the Ricci tensor (i.e. they are unit vectors in the principal directions). We can easily find that the problem of the freedom has an intimate connection with the properties of $\nu_{\alpha}$ 's. Moreover $\nu_{\alpha}$ 's are invariant under coordinate transformations. Therefore, we classify all $V$ 's in terms of $\nu_{\alpha}$ 's as follows:

$V_{\mathrm{I}}$ : The case of 4 simple eigenvalues or, in terms of $\nu_{\alpha}$ 's in (1.10), the case of $\left\{\nu_{1}, \nu_{2}, \nu_{3}, \nu_{4} \neq\right\}$.

$V_{\mathrm{II}}$ : The case of 2 simple eigenvalues and 1 double eigenvalue. $V_{\mathrm{II}}$ 's are further classified into the following four types:

$$
V_{\mathrm{II} 1}\left\{\begin{array}{ccc}
V_{\mathrm{II} a}: & \text { The case of } & \left\{\nu_{2}=\nu_{3} ;\left(\nu_{1}, \nu_{2}, \nu_{4} \neq\right)\right\} . \\
V_{\mathrm{II} b}: \quad " \quad & \left\{\nu_{1}=\nu_{2} ;\left(\nu_{1}, \nu_{3}, \nu_{4} \neq\right)\right\} \text { or }\left\{\nu_{1}=\nu_{3} ;\right. \\
& \left.\left(\nu_{1}, \nu_{2}, \nu_{4} \neq\right)\right\} .
\end{array}\right.
$$




$$
V_{\mathrm{II} 2}\left\{\begin{array}{rr}
V_{\mathrm{II} c}: \text { The case of } & \left\{\nu_{3}=\nu_{4} ;\left(\nu_{1}, \nu_{2}, \nu_{4} \neq\right)\right\} \text { or }\left\{\nu_{2}=\nu_{4} ;\right. \\
& \left.\left(\nu_{1}, \nu_{3}, \nu_{4} \neq\right)\right\} . \\
V_{\mathrm{II} d}: \quad, \quad, \quad\left\{\nu_{1}=\nu_{4} ;\left(\nu_{1}, \nu_{2}, \nu_{3} \neq\right)\right\} .
\end{array}\right.
$$

$V_{\mathrm{III}}$ : The case of 1 simple eigenvalue and 1 triple eigenvalue. $V_{\mathrm{III}}$ 's are further classified into the following three types:

$$
\begin{aligned}
& V_{\mathrm{III} 1}=V_{\mathrm{III} a} ; \text { The case of }\left\{\nu_{1}=\nu_{2}=\nu_{3} \neq \nu_{4}\right\} \text {. } \\
& V_{\mathrm{III} 2}\left\{\begin{array}{llll}
V_{\mathrm{III} b}: & \cdots & \cdots & \left\{\nu_{1}=\nu_{2}=\nu_{4} \neq \nu_{3}\right\} \\
V_{\mathrm{IIIc}}: & , & , & \left\{\nu_{1} \neq \nu_{2}=\nu_{3}=\nu_{4}\right\} .
\end{array}\right.
\end{aligned}
$$

$V_{\mathrm{IV}}$ : The case of 2 double eigenvalues. $V_{\mathrm{IV}}$ 's are further classified into the following two types:

$$
\left\{\begin{array}{c}
V_{\mathrm{IV} a}: \text { The case of }\left\{\nu_{1}=\nu_{2} \neq \nu_{3}=\nu_{4}\right\} \\
V_{\mathrm{IV} b}: \quad \text { or } \quad\left\{\nu_{1}=\nu_{3} \neq \nu_{2}=\nu_{4}\right\} .
\end{array}\right.
$$

$V_{\mathrm{v}}$ : The case of 1 quadruple eigenvalue, i.e. the case of $\left\{\nu_{1}=\nu_{2}=\nu_{3}\right.$ $=\nu$.

Here we shall add a proposition which is closely connected with the classifications in the above:

$\mathrm{P}_{\mathrm{RO} O \mathrm{P} I T I O N}$ 2.1. Let $V$ be non-Minkowskian, i.e. non-flat. Then we can discriminate invariantly $\stackrel{1}{\mathfrak{u}_{i}}$ from $\stackrel{2}{u_{i}}$ (or $\stackrel{3}{u_{i}}$ ).

PROOF. Both $\stackrel{1}{u_{i}}$ and $\stackrel{2}{u_{i}}$ are space-like unit eigenvectors of $K_{i}^{j}$. We have $\stackrel{1}{u}^{i} \nabla_{i}{ }_{i}^{1} u_{j}=0$ and $\stackrel{2}{u^{i} \nabla_{i}} \stackrel{2}{u_{j}}=-\lambda_{2} u_{j} \neq 0$, when $\lambda_{2} \neq 0$. Next, if $\lambda_{2}=0$, we have $\nabla_{i} \stackrel{2}{u_{j}}=0$ and $\nabla_{i} u_{j}=-\lambda_{3} u_{i} u_{j}+\lambda_{4} u_{i} u_{j} \neq 0$. (Note that $V$ is flat when $\lambda_{2}=\lambda_{3}=\lambda_{4}=0$ holds.) Similarly, we can distinguish invariantly $\stackrel{1}{u_{i}}$ from $\stackrel{3}{u_{i}}$. Q.E.D.

In the following sections, we shall make clear the actual methods of classifying the eleven types of $V$ 's listed above when the fundamental tensors are given.

\section{§3. Some preparatory propositions, 1 .}

It is evident that $V$ 's of type $V_{\mathrm{I}}$ or $V_{\mathrm{V}}$ are completely characterized by the condition $\left\{\nu_{1}, \nu_{2}, \nu_{3}, \nu_{4} \neq\right\}$ or $\left\{\nu_{1}=\nu_{2}=\nu_{3}=\nu_{4}\right\}$ respectively, and that the problem of the invariant classification is out of the question for $V$ 's of these 
two types. The problem is important when we consider the classifications of $V_{\text {II }}$ 's, $V_{\text {III }}$ 's and $V_{\text {IV }}$ 's. In this section we first prove the following proposition which will play an important role in the theory of classification:

Proposition 3.1. Let (1.1) be the line element of a V. If we assume that all six eigenvalues of $\boldsymbol{K}_{A}^{\cdot B}\left(\equiv K_{i j}^{\cdot m n} ; A \equiv(i j), B \equiv(m n) ; 1 \equiv(12), 2 \equiv(13)\right.$, $\cdots, 6 \equiv(34) ; A, B=1,2, \cdots, 6)$ are constants, then the possible $(\beta, \gamma, \delta)$ and the corresponding $(B, C, D)$ are given by the following four types:

(I) When $\beta \gamma \delta \neq 0$, we have

$$
\begin{gathered}
\beta=2 p_{2}, \quad \gamma=2 p_{3}, \quad \delta=2 p_{4}, \\
B=c_{2} \exp \left(2 p_{2} x\right), \quad C=c_{3} \exp \left(2 p_{3} x\right), \quad D=c_{4} \exp \left(2 p_{4} x\right),
\end{gathered}
$$

where (and throughout the remainder of the paper) $c_{2}, c_{3}$ and $c_{4}$ are arbitrary positive constants, and p's arbitrary non-vanishing constants. In this case, we have

$$
\lambda_{1 a}=\left(p_{a}\right)^{2}, \quad \lambda_{a b}=p_{a} p_{b} .
$$

When and only when $p_{2}=p_{3}=p_{4}$ holds, the $V$ is $S(A)$.

(II) When one of $(\beta, \gamma, \delta)$ is 0 and the remaining two are non-zero, i.e. when one of $\left(\mathrm{II}_{2}\right)(\beta=0, \gamma \delta \neq 0),\left(\mathrm{II}_{3}\right)(\gamma=0, \delta \beta \neq 0)$ and $\left(\mathrm{II}_{4}\right)(\delta=0, \beta \gamma \neq 0)$ holds, we have, for example, for $\left(\mathrm{II}_{4}\right)$

$\left(\mathrm{II}_{4 a}\right): \quad(3.1),(3.2),(3.3)$ with $\left(p_{4}=0, p_{2} p_{3} \neq 0\right)$,

$\left(\mathrm{II}_{4 b}\right)$ :

$$
\begin{aligned}
& \beta=2 p\left(a e^{p x}-b e^{-p x}\right)\left(a e^{p x}+b e^{-p x}\right)^{-1}, \\
& \gamma=2 p\left(a e^{p x}+b e^{-p x}\right)\left(a e^{p x}-b e^{-p x}\right)^{-1}, \quad \delta=0 ;
\end{aligned}
$$

$$
B=c_{2}\left(a e^{p x}+b e^{-p x}\right)^{2}, \quad C=c_{3}\left(a e^{p x}-b e^{-p x}\right)^{2}, \quad D=c_{4},
$$

or $\left(\mathrm{II}_{4 b^{\prime}}\right)$ :

$$
\begin{aligned}
& \beta=2 p(a \cos p x-b \sin p x)(a \sin p x+b \cos p x)^{-1}, \\
& \gamma=-2 p(a \sin p x+b \cos p x)(a \cos p x-b \sin p x)^{-1}, \quad \delta=0 ;
\end{aligned}
$$

(3. $\left.5^{\prime}\right) \quad B=c_{2}(a \sin p x+b \cos p x)^{2}, \quad C=c_{3}(a \cos p x-b \sin p x)^{2}, \quad D=c_{4}$, where $p(\neq 0), a$ and $b$ are arbitrary constants, which do not satisfy $a=b$ $=0$. We have for $\left(\mathrm{II}_{4 b}\right)$ and $\left(\mathrm{II}_{4 b^{\prime}}\right)$

$$
\lambda_{14}=\lambda_{24}=\lambda_{34}=0, \quad \lambda_{12}=\lambda_{13}=\lambda_{23}= \pm p^{2} \quad(\equiv P),
$$

where + and - correspond to $\left(\mathrm{II}_{4 b}\right)$ and $\left(\mathrm{II}_{4 b^{\prime}}\right)$ respectively. Similar results hold for $\left(\mathrm{II}_{2}\right)$ and $\left(\mathrm{II}_{3}\right)$. The $V$ in $\left(\mathrm{II}_{4 b}\right)$ or $\left(\mathrm{II}_{4 b^{\prime}}\right)$ is nothing but $S(C)$, and the 
corresponding ones in $\left(\mathrm{II}_{2 b}\right),\left(\mathrm{I}_{2 b^{\prime}}\right),\left(\mathrm{II}_{3 b}\right)$ and $\left(\mathrm{II}_{3 b^{\prime}}\right)$ are of the same character except the signature, i.e. each $V$ is a direct product of a straight line and a 3-dimensional space of constant curvature.

(III) When two of $(\beta, \gamma, \delta)$ are 0 and the remaining one is non-zero, i.e. when one of $\left(\mathrm{III}_{2}\right)(\beta \neq 0, \gamma=\delta=0),\left(\mathrm{III}_{3}\right)(\gamma \neq 0, \delta=\beta=0)$ and $\left(\mathrm{III}_{4}\right)(\delta \neq 0$, $\beta=\gamma=0)$ holds, we have, for example, for $\left(\mathrm{III}_{4}\right)$

$\left(\mathrm{III}_{4 a}\right)$ :

$$
\begin{aligned}
& \beta=\gamma=0, \quad \delta=2(x+c)^{-1}, \\
& B=c_{2}, \quad C=c_{3}, \quad D=c_{4}(x+c)^{2},
\end{aligned}
$$

where $c$ is an arbitrary constant. In this case, we have

$$
\lambda_{1 a}=\lambda_{a b}=0, \text { and accordingly } \boldsymbol{K}_{A}^{\cdot B}=0 \text {, i.e. } K_{i j}^{\cdot m n}=0,
$$

which means that the $V$ is $S(B)$.

$\left(\mathrm{III}_{4 b}\right)$ :

$$
\begin{aligned}
& \beta=\gamma=0, \quad \delta=2 p\left(a e^{p x}-b e^{-p x}\right)\left(a e^{p x}+b e^{-p x}\right)^{-1}, \\
& B=c_{2}, \quad C=c_{3}, \quad D=c_{4}\left(a e^{p x}+b e^{-p x}\right)^{2}
\end{aligned}
$$

$\left(\mathrm{III}_{4 b^{\prime}}\right)$ :

$$
\begin{aligned}
& \beta=\gamma=0, \quad \delta=2 p(a \cos p x-b \sin p x)(a \sin p x+b \cos p x)^{-1}, \\
& B=c_{2}, \quad C=c_{3}, \quad D=c_{4}(a \sin p x+b \cos p x)^{2},
\end{aligned}
$$

where $p, a$ and $b$ are of the same meanings as in $\left(\mathrm{II}_{4 b}\right)$ and $\left(\mathrm{II}_{4 b^{\prime}}\right)$. In this case, we have

$$
\lambda_{12}=\lambda_{13}=\lambda_{23}=\lambda_{24}=\lambda_{34}=0, \quad \lambda_{14}= \pm p^{2} \equiv P .
$$

Similar results hold for $\left(\mathrm{III}_{2}\right)$ and $\left(\mathrm{III}_{3}\right)$.

(IV) When all of $(\beta, \gamma, \delta)$ vanish, we have

$$
\beta=\gamma=\delta=0 ; B=c_{2}, \quad C=c_{3}, \quad D=c_{4} .
$$

Proof. The proof is easy if we use the relations:

$$
\begin{aligned}
& 4 \lambda_{12}=2 \beta^{\prime}+\beta^{2}, \quad 4 \lambda_{13}=2 \gamma^{\prime}+\gamma^{2}, \quad 4 \lambda_{14}=2 \delta^{\prime}+\delta^{2}, \\
& 4 \lambda_{34}=\gamma \delta, \quad 4 \lambda_{24}=\beta \tilde{\delta}, \quad 4 \lambda_{23}=\beta \gamma,
\end{aligned}
$$

which are obtained from (1.3), (1.4) and (1.5). Hence we omit it. (See §7 below).

Here the notations $S(A), S(B)$ and $S(C)$ denote respectively de Sitter's space-time (or geometrically, the space-time of constant curvature), Min- 
kowski's space-time (or geometrically, the flat space-time) and Einstein's space-time. These notations were introduced in [2], and we use them only for brevity's sake. As is well-known, both $S(A)$ and $S(C)$ played important roles in the early stage of relativistic cosmology as models of the universe.

We shall denote in the following by $V_{0}$ a $V$ whose six eigenvalues of $\boldsymbol{K}_{A}^{B}$ are all constants, and further by $S(\bar{C})$ the $V$ which belongs to $\left(\mathrm{II}_{2 b}\right)$, $\left(\mathrm{II}_{2 b^{\prime}}\right),\left(\mathrm{II}_{3 b}\right)$ or $\left(\mathrm{II}_{3 b^{\prime}}\right)$ and corresponds to $S(C)$. In more detail, $(B, C, D)$ of an $S(\overline{\boldsymbol{C}})$ are obtained from $(3.5)$ or $\left(3.5^{\prime}\right)$ by interchanging, for example, $C$ with $D$.

It should be noted here that the cases obtained from (I) by putting, for example, $\left(p_{2} \neq 0, p_{3}=p_{4}=0\right)$ are included in $\left(\mathrm{III}_{\rho b}\right)$ or $\left(\mathrm{III}_{\rho b^{\prime}}\right),(\rho=2,3,4)$, and that we have no need of dealing with such cases separately.

Next, we proceed to the consideration of the relation between the $V$ 's obtained in the above proposition, and to the classification of $V$ 's in terms of the four eigenvalues of the Ricci tensor given in $\$ 2$. By examining in detail the $V$ 's in the classification table of $\S 2$ from the standpoint of the proposition, we have

Proposition 3. 2. $V_{0}$ 's belonging to $V_{\mathrm{I}}, V_{\mathrm{II}}, \cdots, V_{\mathrm{V}}$ respectively are given by the following table:

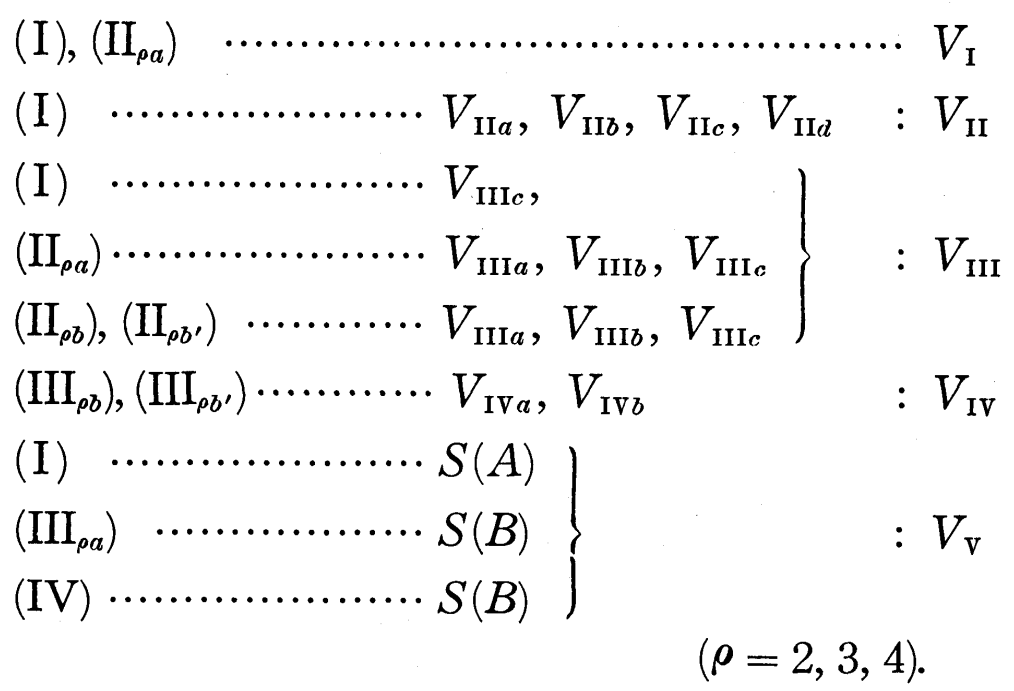

The meanings of the table will easily be understood. More detailed results have been obtained. For example, $V_{\mathrm{III} a}$ belonging to $\left(\mathrm{II}_{\rho a}\right)$ is possible only for $\rho=4$. But we omit them for brevity's sake.

\section{$\S 4$. Invariant classification of $V_{\mathrm{II}}^{\text {' }} \mathrm{s}$.}

A $V_{\mathrm{II}}$ is characterized by $\{2$ simple eigenvalues and 1 double eigenvalue $\}$ of the Ricci tensor. As is seen in $\S 2, V_{\mathrm{II}}$ 's are classified into two classes 
$V_{I 11}$ and $V_{I 12}$, and each class into two subclasses. When all eigenvectors corresponding to the double eigenvalue are space-like, the $V_{\mathrm{II}}$ is $V_{\mathrm{III}}$. In this case, eigenvectors corresponding to the two simple eigenvalues are spacelike and time-like respectively. On the other hand, when all eigenvectors corresponding to the simple eigenvalues are space-like, the $V_{\mathrm{II}}$ is $V_{\mathrm{II} 2}$, and in this case, the eigenvectors corresponding to the double eigenvalue can be space-like or null or time-like.

Now we shall study how to classify invariantly $V_{\mathrm{II} a}$ and $V_{\mathrm{II} b}$, and then $V_{\text {II } c}$ and $V_{\text {II } d}$.

First we consider $V_{\text {III }}$ denoting by $\nu$ the simple eigenvalue to which the unit space-like eigenvector $u_{i}$ corresponds. Take any $V_{I I a}$, and it is evident that we have $\nu=\nu_{1}$ and $u_{i}=\varepsilon u_{i},\left(\varepsilon^{2}=1\right)$, which is evidently a gradient. On the other hand, if the $V_{\mathrm{II} 1}$ is $V_{\mathrm{II} b}$, we have $\nu=\nu_{3}\left(\right.$ or $\left.\nu_{2}\right)$ and $u_{i}=\varepsilon u_{i}$ (or $\varepsilon u_{i}$ ). Further, the condition that the $\stackrel{3}{u_{i}}$ (or $\stackrel{2}{u_{i}}$ ) be a gradient is given by $\lambda_{3}=r=0$ (or $\lambda_{2}=\beta=0$ ), from which we have $\nu_{3}=0$ (or $\left.\nu_{2}=0\right)$. Thus we can conclude that, when $u_{i}$ of a $V_{\mathrm{II} 1}$ is not a gradient, the $V_{\mathrm{II} 1}$ is $V_{\mathrm{II} b}$, and further, when $u_{i}$ is a gradient and $\nu \neq 0$, the $V_{\mathrm{II} 1}$ is $V_{\mathrm{II} a}$.

Next we consider the case in which $u_{i}$ is a gradient and $\nu=0$. Then it is easy to see that such a $V_{\text {III }}$ cannot be a $V_{0}$. In other words, at least one of six eigenvalues of $\boldsymbol{K}_{A}^{B}$ is non-constant. If we denote by $\lambda$ any of such non-constant eigenvalues, then it is evident that when $\nabla_{i} \lambda$ is proportional to the $u_{i}$, the $V_{\mathrm{II} 1}$ is $V_{\mathrm{II} a}$, and otherwise it is $V_{\mathrm{II} b}$. These results can be written in a table as follows:

$$
\begin{aligned}
& u_{i} \text { is not a gradient .......................... } V_{\mathrm{II} b}
\end{aligned}
$$

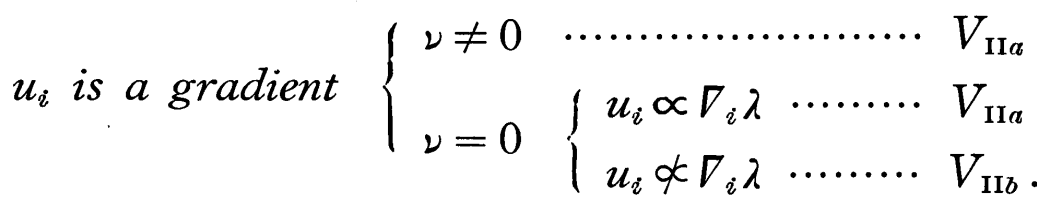

Now we proceed to the classification of $V_{\text {II } 2}$ 's. Let $\left(\nu, u_{i}\right)$ and $\left(\nu^{\prime}, u_{i}^{\prime}\right)$ be two sets of a simple eigenvalue and the corresponding unit space-like eigenvector. It is evident that when both $u_{i}$ and $u_{i}^{\prime}$ are not gradients, the $V_{\text {II2 }}$ is $V_{\text {IId }}$. Next we consider the case in which $u_{i}$ is a gradient but $u_{i}^{\prime}$ is not. If the $V_{\text {II2 }}$ is $V_{\text {IIld }}$, we have $\nu=0$ from (1.7). Thus, when $\nu \neq 0$, the $V_{\mathrm{II} 2}$ is $V_{\mathrm{II} c}$. When $u_{i}$ is a gradient, $\nu=0$ and $u_{i}^{\prime}$ is not a gradient, we can find, just as in the case of $V_{\text {II1 }}$, that any $V_{\text {II2 }}$ satisfying these conditions cannot be $V_{0}$, and that the $V_{\text {II } 2}$ is $V_{\text {IIc }}$ or $V_{\text {II } d}$ according as $u_{i} \propto \nabla_{i} \lambda$ or $u_{i}^{\prime} \propto \nabla_{i} \lambda$ respectively. Lastly we deal with the case in which both $u_{i}$ and $u_{i}^{\prime}$ are not gradients. We can easily see that any $V_{\text {IId }}$ cannot satisfy this 
condition, and hence the $\mathrm{V}_{\mathrm{II} 2}$ is $V_{\mathrm{II} c}$. Corresponding to the table of $V_{\mathrm{III}}$, we have:

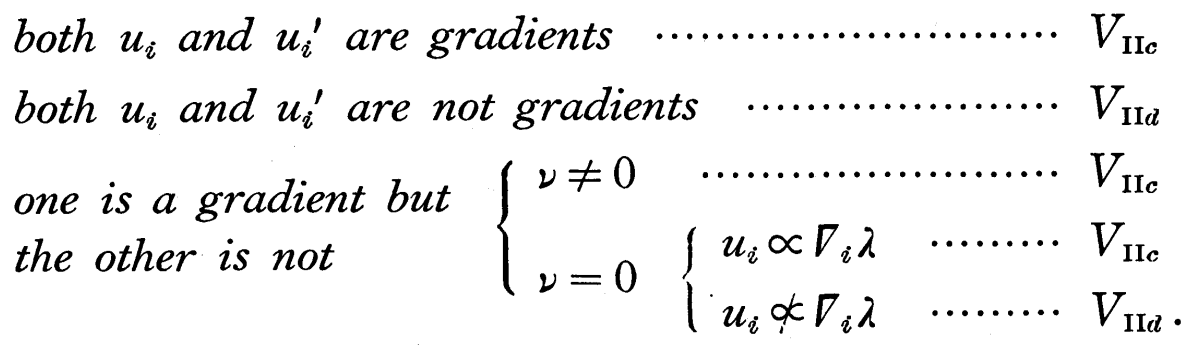

Lastly it should be noted that the $V_{\text {II }}$ 's of the four types dealt with in this section really exist and that it is not difficult to give some examples of the actual forms of their line elements.

\section{§5. Some preparatory propositions, 2 .}

We proceed to the classification of $V_{\text {III }}$ 's. $V_{\text {III }}$ is characterized by $\{1$ simple eigenvalue and 1 triple eigenvalue $\}$. Further $V_{\mathrm{III}}\left(=V_{\mathrm{III} a}\right)$ is characterized by that the eigenvectors corresponding to the simple eigenvalue is time-like, while $V_{\text {III } 2}$ (i.e. $V_{\text {III }}$ or $V_{\text {IIIc }}$ ) is by that they are space-like. The reason why we distinguish $V_{\mathrm{III} b}$ from $V_{\mathrm{III} c}$ lies in Proposition 2.1.

First we prove some propositions which are necessary when we deal with the freedom of c.s. of $V_{\text {III } a}$.

Proposition 5.1. Let $D$ in the coordinate system of (1.1) satisfies $D^{\prime}=0$ (and accordingly, $\delta=0$ ) and $\nu^{\prime} s$ be of the form $\nu_{1}=\nu_{2}=\nu_{3} \neq \nu_{4}=0$. Then $\nu_{1}$ must be a constant $(\neq 0)$.

Proof. From the actual expression of $\nu$ 's given in $\S 1$ and the assumption, we have

$$
-2 \nu_{1}=\beta \gamma, \quad 2 \beta^{\prime}+\beta^{2}=\beta \gamma=2 \gamma^{\prime}+\gamma^{2},
$$

from which we can easily obtain $\nu_{1}^{\prime}=0$. Q.E. D.

Proposition 5.2. The line element of the $V_{\mathrm{III} \text { stated in Proposition }}$ 5.1 is reducible to the form

$$
d s^{2}=-d x^{2}-B d y^{2}-C d z^{2}+d t^{2},
$$

where $B$ and $C$ are functions of $x$ given by

(3.5) when $\nu_{1}<0$,

(3. $\left.5^{\prime}\right)$ when $\nu_{1}>0$.

Corresponding to (5.3) or $\left(5.3^{\prime}\right)$, we have respectively

$$
\nu_{1}=-2 p^{2},\left(5.4^{\prime}\right) \quad \nu_{1}=2 p^{2} .
$$


Note that we have $K=3 \nu_{1}=\mp 6 p^{2}$ respectively.

Proof. It is evident that $\beta \neq 0$. Hence we have from (5.1)

$$
2 \beta^{\prime} / \beta+\beta-\gamma=0, \text { i.s. } 2 \beta^{\prime} / \beta+B^{\prime} / B-C^{\prime} / C=0,
$$

the integration of which gives $B^{\prime} / 2 \sqrt{B}=e_{1} \sqrt{C}$. Similarly we have $C^{\prime} / 2 \sqrt{C}$ $=e_{2} \sqrt{B}$. Here $e_{1}$ and $e_{2}$ are arbitrary non-vanishing constants. When $e_{1} e_{2}>0$, putting $\sqrt{e_{1} e_{2}}=p$ and integrating these equations, we obtain

$$
\sqrt{B}=m_{1} e^{p x}+m_{2} e^{-p x}, \quad \sqrt{C}=\sqrt{e_{2} / e_{1}}\left(m_{1} e^{p x}-m_{2} e^{-p x}\right), \quad\left(\beta \gamma=4 p^{2}\right),
$$

where $m_{1}$ and $m_{2}$ are arbitrary constants which do not satisfy $m_{1}=m_{2}=0$. Then it is evident that we have (5.3) by a simple transformation. On the other hand, when $e_{1} e_{2}<0$, we can similarly obtain $\left(5.3^{\prime}\right)$ by putting $\sqrt{-e_{1} e_{2}}$ $=p$. The remaining part is evident. Q.E.D.

Let $[K]$ be the c.s. for which the coordinate system of (5.2) with (5.3) (or $\left(5.3^{\prime}\right)$ ) is standard. Then we have from (1.3)

$$
\lambda_{4}=0, \quad \lambda_{2} \lambda_{3}=\beta r / 4= \pm p^{2} \equiv P,
$$

from which we have

Proposition 5.3. If we use $[K]$, the classification of $(5.3)$ and $\left(5.3^{\prime}\right)$, or equivalently, that of (5.4) and (5.4'), is equivalent to

$$
\lambda_{2} \lambda_{3}>0 \text {, }
$$

$$
\lambda_{2} \lambda_{3}<0 \text {. }
$$

Further we have

PROPOSITION 5.4. In the primed case, we cannot have $\lambda_{2}=\lambda_{3}$ so far as we are dealing with real quantities. In the unprimed case, on the other hand, a necessary and sufficient condition for $\lambda_{2}=\lambda_{3}$ is given by $(a \neq 0, b=0)$ or $(a=0, b \neq 0)$, and it holds that $\lambda_{2}=\lambda_{3}=p$ in the former case and $\lambda_{2}=\lambda_{3}$ $=-p$ in the latter.

Note that in the primed case, if we admit complex quantities, the condition for $\lambda_{2}=\lambda_{3}$ is given by $a= \pm i b$, and again we have $\lambda_{2}=\lambda_{3}=$ const. $\neq 0$.

For $V_{\text {III } a}$ of Proposition 5.1, we have from (1.5)

$$
\lambda_{12}=\lambda_{13}=\lambda_{23}=P, \quad \lambda_{14}=\lambda_{24}=\lambda_{34}=0 .
$$

The $V_{\text {III } a}$ under consideration is nothing but the $S(C)$ as is elucidated in §3. It is easy to see from (5.1), etc. that it satisfies

$$
K_{\boldsymbol{\rho}_{\sigma}}^{\mu^{\mu \nu}}=\left(\nu_{1} / 2\right)\left(\delta_{\rho}^{\mu} \delta_{\sigma}^{\nu}-\delta_{\sigma}^{\mu} \delta_{\rho}^{\nu}\right), \quad(\rho, \sigma, \cdots=1,2,3),
$$

where $\nu_{1}=-2 P$. (5.10) together with (5.2) shows that the 3-dimensional space of $(x, y, z)$ is of constant curvature. Here it should be noted that we 
use the notation $S(C)$ irrespectively of whether $\nu_{1}=-2 p^{2}$ or $\nu_{1}=2 p^{2}$ holds. The most familiar form of its line element in relativistic theories is

$$
d s^{2}=-\left(1+r^{2} / 4 \mathrm{R}^{2}\right)^{-2}\left(d x^{2}+d y^{2}+d z^{2}\right)+d t^{2},
$$

where $r=\sqrt{x^{2}+y^{2}+z^{2}}$ and $1 / R^{2}$ is a constant. It is easy to see that the relation between $R^{2}$ and $P\left(= \pm p^{2}\right)$ is given by

$$
1 / R^{2}=P=-\nu_{1} / 2 \text {. }
$$

From the results obtained above, we have

Proposition 5. 5. In $S(C)$ of the unprimed type, $(i)$ we have c.s. satisfying $\lambda_{2}=\lambda_{3}$ and those satisfying $\lambda_{2} \neq \lambda_{3}$, (ii) the former satisfy either $\lambda_{2}=\lambda_{3}=p$ or $\lambda_{2}=\lambda_{3}=-p$, and (iii) when $\lambda_{2}$ (or $\lambda_{3}$ ) is a constant (accordingly $\lambda_{3}\left(\right.$ or $\left.\lambda_{2}\right)$ is also a constant), we have $\lambda_{2}=\lambda_{3}$.

Proof. We have only to prove (iii). Take the standard coordinate system for the c.s. If we put $\lambda_{2}=p_{2}$ and $\lambda_{3}=p_{3}$, where $p$ 's are constants satisfying $p_{2} p_{3}=p^{2}$, then from $\lambda_{2}=-\beta / 2$ and $\lambda_{3}=-\gamma / 2$, we have $B=c_{2} \exp$ $\left(-2 p_{2} x\right)$ and $C=c_{3} \exp \left(-2 p_{3} x\right)$, where $c$ 's are arbitrary positive constants. From the relations $-4 \nu_{1}=\beta^{2}+\gamma^{2}=\beta^{2}+\beta \gamma=\gamma^{2}+\beta \gamma$, we have $\beta=\gamma, p_{2}=p_{3}$ and $\lambda_{2}=\lambda_{3}$ in turn. This result is seen also from the fact that when $\lambda_{2} \neq \lambda_{3}$, both $\lambda_{2}$ and $\lambda_{3}$ cannot be constants. Q.E.D.

Now we shall add some propositions concerning $S(C)$. The meaning of the results so far obtained will be understood more deeply by these propositions. A parallel vector field ('field' will be omitted hereafter) $v_{i}$ is defined by

$$
\nabla_{i} v_{j}=0
$$

from which follows $v_{i}=\partial_{i} v$. Now we consider the general $V$. In the coordinate system of (1.1), (5.13) becomes by virtue of (2.2) of [1]

$$
\begin{aligned}
\partial_{11} v & =\partial_{23} v=\partial_{24} v=\partial_{34} v=\left\{\partial_{12}-(\beta / 2) \partial_{2}\right\} v \\
& =\left\{\partial_{13}-(\gamma / 2) \partial_{3}\right\} v=\left\{\partial_{14}-(\delta / 2) \partial_{4}\right\} v=\left\{\partial_{22}+\left(B^{\prime} / 2\right) \partial_{1}\right\} v, \\
& =\left\{\partial_{33}+\left(C^{\prime} / 2\right) \partial_{1}\right\} v=\left\{\partial_{44}-\left(D^{\prime} / 2\right) \partial_{1}\right\} v=0 .
\end{aligned}
$$

By solving (5.14), we can easily obtain (cf. Proposition 3.1)

Proposition 5. 6. When $\beta \gamma \delta \neq 0$, the $V$ admits no parallel vector. (II) when one of $\left(\mathrm{II}_{2}\right)(\beta=0, \gamma \delta \neq 0),\left(\mathrm{II}_{3}\right)(\gamma=0, \delta \beta \neq 0)$ and $\left(\mathrm{II}_{4}\right)(\delta=0, \beta \gamma \neq 0)$ holds, we have one and only one parallel vector $v_{i}$. It is $c \delta_{i}^{2}, c \delta_{i}^{3}$ (space-like), and $c \delta_{i}^{4}$ (time-like) respectively. Here $c$ is a non-vanishing arbitrary constant. (III) When one of $\left(\mathrm{III}_{2}\right)(\gamma=\delta=0, \beta \neq 0),\left(\mathrm{III}_{3}\right)(\delta=\beta=0, \gamma \neq 0)$ and $\left(\mathrm{III}_{4}\right)(\beta=\gamma$ 
$=0, \delta \neq 0)$ holds and the $V$ is non-flat, we have two linearly independent (with constant coefficients) parallel vectors. They are $c \delta_{i}^{3}+c^{\prime} \delta_{i}^{4}, c \delta_{i}^{4}+c^{\prime} \delta_{i}^{2}$ (space-like, null, time-like) and $c \delta_{i}^{2}+c^{\prime} \delta_{i}^{3}$ (space-like) respectively. Here $c$ and $c^{\prime}$ are arbitrary constants which do not satisfy $c=c^{\prime}=0$. When the $V$ is flat, i.e. when (3.7) or a similar relation holds, we have evidently four linearly independent parallel vectors. In other words, we have parallel vectors in any direction at any point. (IV) When $\beta=\gamma=\delta=0$ holds, the $V$ is flat, and we have four linearly independent parallel vectors.

Consequently, we find that, for example, there exists no $V$ admitting three parallel vectors, which is also seen from the well-known theorem of Walker [3].

Evidently $S(C)$ belongs to the type $\left(\mathrm{II}_{4}\right)$ and admits only one parallel vector which is time-like. (See also [2].) We have further

PROPOSITION 5. 7. $V_{\text {III }}$ (more generally, $V$ ) which satisfies $\delta \neq 0$ in the coordinate system of $(1.1)$ is not $S(C)$.

PROPOSITION 5.8. $S(C)$ cannot admit a c.s. satisfying $\lambda_{2} \lambda_{3} \lambda_{4} \neq 0$.

\section{§6. Invariant classification of $V_{\mathrm{III} 2}$ 's.}

Let $V$ be a $V_{\mathrm{III} 2}$, i.e. $V_{\mathrm{III} b}$ or $V_{\mathrm{III} c}$. We consider the problem of classifying invariantly $V_{\mathrm{III} b}$ and $V_{\mathrm{III} c}$. Let $\nu$ and $\nu^{\prime}$ be the simple and triple eigenvalues respectively. In any $V_{\text {III2 }}$, the unit eigenvector $u_{i}$ corresponding to $\nu$ is space-like, and further we have

PROPOSITION 6.1. In $V_{\mathrm{III}}$, the unit eigenvector $v_{i}$ is a gradient. Accordingly, $V_{\mathrm{III} 2}$ is $V_{\mathrm{III}}$ if $u_{i}$ is not a gradient.

The proof is evident, since $u_{i}$ is nothing but $\varepsilon u_{i}\left(\varepsilon^{2}=1\right)$.

PROposition 6. 2. Assume that $u_{i}$ in a $V_{\mathrm{III} 2}$ be a gradient. Then the $V_{\mathrm{III} 2}$ is $V_{\mathrm{IIIb}}$ or $V_{\mathrm{III} c}$ according as $u_{i}$ is a parallel field or not.

PROOF. The proof is easy if we use Proposition 5.6 and the fact that the condition $\stackrel{3}{u_{i}}=\sqrt{C} \delta_{i}^{3}$ be a gradient (i. e. $\nabla_{[i} u_{j]}=0$ ) is given by $\lambda_{3}=0$ (i. e. $\gamma=0)$ and that we have $\nabla_{i} u_{j}=0$ in this case.

In connection with this proposition, we add the following:

PROPOSITION 6. 3. $V_{\mathrm{III} 2}$ cannot admit a c.s. satisfying $\lambda_{2}=\lambda_{3}=0$.

PRoposition 6. 4. Any c.s. of the $V_{\mathrm{III}}$ stated in Proposition 6.2 satisfies either $\left(\lambda_{2} \neq 0, \lambda_{3}=0\right)$ or $\left(\lambda_{2}=0, \lambda_{3} \neq 0\right)$. For this $V_{\mathrm{IIIb}}$, we have $\nu=0$.

PROPOSITION 6.5. When a c.s. of $V_{\text {IIIc }}$ satisfies either $\left(\lambda_{2} \neq 0, \lambda_{3}=0\right)$ or $\left(\lambda_{2}=0, \lambda_{3} \neq 0\right)$, we have $\nu^{\prime}=0$. 
The proofs of these propositions are easy, so we omit them.

From the discussions of the last and the present sections, we can conclude that when a $V_{\text {III }}$ is given in any coordinate system, we can determine whether it is $V_{\mathrm{III} a}$ or $V_{\mathrm{III} b}$ or $V_{\mathrm{III} c}$. The classifications are independent of the coordinate system.

REMARK. As is seen in $\S 4$, the classification of $V_{\mathrm{II}}$ 's is easy if we use Proposition 3. 1. However, it is not so easy to classify $V_{\text {III }}$ 's by using the same proposition. The reason is that we have many $V_{\text {III2 }}$ 's belonging to $V_{0}$.

Now we shall write down some propositions corresponding to those in $\S 5$. These propositions will be of use when we consider the freedom of c. s. in $V_{\mathrm{IIIb}}$. The proofs are similar to those in $\$ \mathbf{5}$.

Proposition 6.6. Let $C$ in the coordinate system of (1.1) satisfy $C^{\prime}=0$ (i.e. $\left.\gamma=0\right)$ and $\nu^{\prime}$ 's be of the form $\nu_{1}=\nu_{2}=\nu_{4} \neq \nu_{3}=0$. Then $\nu_{1}\left(\right.$ i.e. $\left.\nu^{\prime}\right)$ must be a non-vanishing constant.

Proposition 6.7. The line element of the $V_{\mathrm{IIIb}}$ stated above is reducible to the form

$$
d s^{2}=-d x^{2}-B d y^{2}-d z^{2}+D d t^{2},
$$

where $B$ and $D$ are functions of $x$ given by

$$
\begin{array}{r}
B=c_{2}\left(a e^{p x}+b e^{-p x}\right)^{2}, \quad C=c_{3}, \quad D=c_{4}\left(a e^{p x}-b e^{-p x}\right)^{2}, \\
\text { when } \nu^{\prime}=-2 p^{2}<0, \\
B=c_{2}(a \sin p x+b \cos p x)^{2}, \quad \begin{aligned}
& C=c_{3}, D=c_{4}(a \cos p x-b \sin p x)^{2}, \\
& \text { when } \quad \nu^{\prime}=2 p^{2}>0 .
\end{aligned}
\end{array}
$$

Here p, c's, $a$ and $b$ are of the same meanings as in Proposition 3.1, and again we have $K=3 \nu^{\prime}=\mp 6 p^{2}$ respectively.

Thus the $V_{\text {IIIb }}$ is nothing but $S(\bar{C})$. We can obtain various results concerning $S(\bar{C})$ similar to those obtained in $\$ 5$ concerning $S(C)$. We have, for example,

$$
\lambda_{2} \lambda_{4}=-\nu^{\prime} / 2= \pm p^{2} \quad(\equiv P),
$$

and we can easily find, just as in the case of $S(C)$, that many kinds of c.s. are possible in $S(\bar{C})$. It goes without saying that we can obtain similar results for the $V_{\text {IIIb }}$ of the type $\nu_{1}=\nu_{3}=\nu_{4} \neq \nu_{2}=0$.

\section{§ 7. Invariant classification of $V_{\mathrm{IV}}$ 's.}

In this section we deal with the case of two double eigenvalues. $V_{\text {IV } a}$ 
and $V_{\text {IV } b}$ belong to this case. In both $V_{\text {IV }}$ 's, eigenvectors corresponding to the one double eigenvalue $\nu$ are space-like and those corresponding to the other eigenvalue $\nu^{\prime}$ are space-like or null or time-like. In $V_{\text {IV } a}$ in which $\nu_{1}=\nu_{2} \neq \nu_{3}=\nu_{4}$ (or $\nu_{1}=\nu_{3} \neq \nu_{2}=\nu_{4}$ ) holds, we have $\nu=\nu_{1}$ and $\nu^{\prime}=\nu_{4}$, while in $V_{\text {IVb }}$ in which $\nu_{1}=\nu_{4} \neq \nu_{2}=\nu_{3}$ holds, we have $\nu=\nu_{2}$ and $\nu^{\prime}=\nu_{4}$.

We can solve the problem of invariant classification of $V_{\text {IV }}$ 's by considering the eigenvalues of $\boldsymbol{K}_{A}^{B}$, i. e. $\lambda_{1 a}$ and $\lambda_{a b}$. First we can prove the following proposition by examining the results of Proposition 3. 1 in detail:

PROPOSITION 7.1. Let a $V_{\mathrm{IV}}$ be $V_{0}$. Then only the following 3 cases are possible: (i) $V_{\mathrm{IV}}$ of type $\left(\mathrm{III}_{2 b}\right)$ or $\left(\mathrm{III}_{2 b^{\prime}}\right):(B, C, D)$ are given by

$$
B=c_{2}\left(a e^{p x}+b e^{-p x}\right)^{2} \text { or }=c_{2}(a \sin p x+b \cos p x)^{2}, \quad C=c_{3}, \quad D=c_{4} .
$$

(ii) $V_{\mathrm{IV}}$ of type $\left(\mathrm{II}_{3 b}\right)$ or $\left(\mathrm{III}_{3 b^{\prime}}\right):(B, C, D)$ are given by the expressions which are obtained from (7.1) by interchanging $B$ with $C$, and $c_{2}$ with $c_{3}$.

The $V_{\mathrm{IV}}$ in $(i)$ or $(i i)$ is $V_{\mathrm{IV} a}$ and we have $\left(\nu=-P, \nu^{\prime}=0\right)$.

(iii) $V_{\mathrm{IV}}$ of type $\left(\mathrm{III}_{4 b}\right)$ or $\left(\mathrm{III}_{4 b^{\prime}}\right):(B, C, D)$ are given by

$$
B=c_{2}, \quad C=c_{3}, \quad D=c_{4}\left(a e^{p x}+b e^{-p x}\right)^{2} \text { or }=c_{4}(a \sin p x+b \cos p x)^{2} .
$$

The $V_{\mathrm{IV}}$ is $V_{\mathrm{IV} b}$ and we have $\left(\nu=0, \nu^{\prime}=-P\right)$.

Here P, $p, c^{\prime}$ s, $a$ and $b$ are of the same meanings as in Proposition 3.1.

We have from this proposition

Proposition 7.2. Let $V_{\mathrm{IV}}$ be $V_{0}$. Then one of $\nu$ and $\nu^{\prime}$ is 0 and the remaining one is a non-vanishing constant $-P$. The $V_{\mathrm{IV}}$ is $V_{\mathrm{IV} a}$ or $V_{\mathrm{IVb}}$ according as $\nu=-P$ or $\nu=0$ respectively.

Next we consider $V_{\text {IV }}$ which is not $V_{0}$. Then at least one of six eigenvalues is not constant. We denote one of such eigenvalues by $\lambda$ using the same notation as in $\S 4$. Then we have from Proposition 7, 1

PRoposition 7.3. Consider a $V_{\mathrm{IV}}$ which is not $V_{0}$. Denote by $u_{i}$ the unit vector proportional to the gradient $\nabla_{i} \lambda_{\text {. Then }} u_{i}$ is a unit eigenvector of $K_{i}^{j}$. The $V_{\mathrm{IV}}$ is $V_{\mathrm{IV} a}$ or $V_{\mathrm{IV} b}$ according as $u_{i}$ corresponds to $\nu$ or $\nu^{\prime}$ respectively.

Here it should be noted that even when two or more eigenvalues of $\boldsymbol{K}_{A}^{\cdot B}$ are not constants, the $u_{i}$ is determined uniquely to within its sign.

Thus the problem of discriminating whether the given $V_{\mathrm{IV}}$ is $V_{\mathrm{IV} a}$ or $V_{\text {IVb }}$ has been completely solved.

\section{§8. Some preparatory propositions, 3 .}

$V_{\mathrm{V}}$ is characterized by the condition $\nu_{1}=\nu_{2}=\nu_{3}=\nu_{4} \equiv \nu$, that is, in $V_{\mathrm{V}}$, 
the Ricci tensor has a quadruple eigenvalue $\nu$. In terms of the usual terminology in Riemannian geometry, $V_{\mathbf{v}}$ belongs to the Einstein space. As is easily seen, any $V$ of constant curvature (i.e. $S(A)$ ), including a flat $V$ (i. e. $S(B))$, is $V_{\mathrm{v}}$.

Proposition 8. 1. In an Einstein $V, \nu$ is a constant, and accordingly $K(=4 \nu)$ is also a constant.

Proof. From the actual expressions of $\nu$ 's in the coordinate system of (1. 1) given in $\S 1$, we find that the condition for the Einstein $V$ is given, in terms of $\lambda$ 's, by

$$
\lambda_{12}=\lambda_{34}, \quad \lambda_{13}=\lambda_{24}, \quad \lambda_{14}=\lambda_{23},
$$

or, in terms of $\beta, \gamma$ and $\delta$, by

$$
2 \beta^{\prime}+\beta^{2}=\gamma \delta, \quad 2 \gamma^{\prime}+\gamma^{2}=\delta \beta, \quad 2 \delta^{\prime}+\delta^{2}=\beta \gamma .
$$

Further we have

$$
\nu=-\left(\lambda_{12}+\lambda_{13}+\lambda_{14}\right)=-(1 / 4)(\beta \gamma+\gamma \delta+\delta \beta), \quad K=4 \nu .
$$

From (8.3) and (8.2), we can obtain $\nu^{\prime}=0$. Q.E.D.

Although we proved directly in the above, it is well-known that the scalar curvature $K$ of an Einstein space is a constant. ([4], p. 93.)

Next we can easily obtain from Proposition 3. 1

Proposition 8.2. The line element of $S(B)$ in the form of $(1.1)$ is given by the following four types:

$$
\begin{aligned}
& \left(\mathrm{III}_{2 a}\right) \quad B=c_{2}(x+c)^{2}, \quad C=c_{3}, \quad D=c_{4}, \\
& \left(\mathrm{III}_{3 a}\right) \quad B=c_{2}, \quad C=c_{3}(x+c)^{2}, \quad D=c_{4}, \\
& \left(\mathrm{III}_{4 a}\right) \quad B=c_{2}, \quad C=c_{3}, \quad D=c_{4}(x+c)^{2}, \\
& (\mathrm{IV}) \quad B=c_{2}, \quad C=c_{3}, \quad D=c_{4},
\end{aligned}
$$

where c's are of the same meanings as those in Proposition 3.1.

It should be noted here that $\beta, \gamma$ and $\delta$ in $\left(\mathrm{III}_{\rho a}\right)$ respectively are not constants. The above proposition can be rewritten in the form:

Proposition 8.3. C.s. of $S(B)$ must satisfy one of the following conditions :

$$
\begin{array}{ll}
\left(\mathrm{III}_{2 a}\right) \quad \lambda_{2} \neq \text { const. }, & \lambda_{3}=\lambda_{4}=0, \\
\left(\mathrm{III}_{3 a}\right) & \lambda_{3} \neq \text { const. }, \quad \lambda_{2}=\lambda_{4}=0, \\
\left(\mathrm{III}_{4 a}\right) & \lambda_{4} \neq \text { const. }, \quad \lambda_{2}=\lambda_{3}=0, \\
\text { (IV) } \quad \lambda_{2}=\lambda_{3}=\lambda_{4}=0 .
\end{array}
$$


Now we consider a non-flat space-time of constant curvature, i. e. $S(A)$. We can easily obtain from Proposition 3.1

Proposition 8.4. The line element of $S(A)$ in the form of $(1.1)$ is given by

$$
B=c_{2} e^{p x}, \quad C=c_{3} e^{p x}, \quad D=c_{4} e^{p x},
$$

where $p= \pm \sqrt{-K / 3}$. Thus we have a real metric of the form (1.1) when and only when $K$ is negative.

Proposition 8.5. C.s. of $S(A)$ must satisfy

$$
\lambda_{2}=\lambda_{3}=\lambda_{4}=-p / 2, \quad\left(p^{2}=-K / 3\right) .
$$

Thus c.s. is real when and only when $K$ is negative.

Evidently, $S(B)$ and $S(A)$ are characterized by that $\boldsymbol{K}_{A}^{B}$ has one sextuple constant eigenvalue. Of course, this eigenvalue is 0 or a non-vanishing constant according as the $V_{\mathrm{v}}$ is $S(B)$ or $S(A)$ respectively. It should especially be noted that there exist some sets of $\left(\lambda_{2}, \lambda_{3}, \lambda_{4}\right)$ whose $\lambda^{\prime}$ 's are not necessarily 0 , for the Minkowski space-time $S(B)$.

\section{§9. Some preparatory propositions, 4.}

In the present and the next sections, we shall integrate (8.2) and determine the actual forms of the line element of $V_{\mathrm{v}}$. As a matter of course, $S(B)$ and $S(A)$ dealt with in the last section are included in the following discussions. From (8.2) and (8.3), we can easily obtain

$$
f^{\prime}+f^{2} / 2+3 K / 2=0,
$$

where we put $f=\beta+\gamma+\delta$. Then if we put $f=2 v^{\prime} / v$, (9.1) becomes

$$
v^{\prime \prime}=-(3 K / 4) v \text {. }
$$

(A) In this section, we consider the case of $K=0$. In this case $g_{i j}$ of the space-time under consideration satisfies the Einstein equation for purely gravitational field:

$$
K_{i j}=0 \text {, }
$$

and the results are especially significant from the physical point of view. Therefore we shall state the results somewhat in detail.

We have from (9.2), or directly from (9.1),

$$
\text { (a) } f=0 \text { or (b) } f=2 M^{-1}, \quad(M \equiv x+c) \text {, }
$$

where $c$ is an arbitrary constant. 
$\left(A_{a}\right)$ In the first place we consider the case of $f=0$. This condition gives $B C D=$ const. When $(i)(\beta, \gamma, \delta \neq)$ holds, we have $\beta \gamma \delta \neq 0$ from (8.2). Further we can easily find that we cannot have such a case so far as we are dealing with real quantities. We can also prove that we cannot have the cases of $\left(i i_{2}\right)(\beta \neq \gamma=\delta),\left(i i_{3}\right)(\gamma \neq \beta=\delta)$ and $\left(i i_{4}\right)(\beta=\gamma \neq \delta)$. If we consider finally the case of $(i i i)(\beta=\gamma=\delta)$, we arrive at $\beta=\gamma=\delta=0$. Therefore we have

Proposition 9. 1. When $K=0$ holds, the only possible solution of (8.2) is given by $\beta=\gamma=\delta=0$, if we assume $f=0$. That is, the $V_{\mathrm{V}}$ is $S(B)$, the line element is given by (IV) of Proposition 8.2, and the c.s. satisfies (IV) of Proposition 8. 3.

$\left(A_{b}\right)$ Now we consider the case in which $f=2 M^{-1} \neq 0$ holds.

(i) We assume $(\beta, \gamma, \delta \neq)$. From (8.2), we have

$$
\beta-\gamma=a_{2} M^{-1}, \quad \gamma-\delta=a_{3} M^{-1}, \quad \delta-\beta=a_{4} M^{-1},
$$

where $a$ 's are arbitrary constants satisfying

$$
a_{2}+a_{3}+a_{4}=0 \text {. }
$$

Then from (8.2) and (9.5), we obtain

(9. 7) $\quad \beta=\left(2+a_{2}-a_{4}\right) M^{-1} / 3, \quad \gamma=\left(2+a_{3}-a_{2}\right) M^{-1} / 3, \quad \delta=\left(2+a_{4}-a_{3}\right) M^{-1} / 3$.

The condition $(\beta, \gamma, \delta \neq)$ is equivalent to $a_{2} a_{3} a_{4} \neq 0$. Using (8.2) again, we have

$$
a_{2}^{2}+{a_{3}}^{2}+a_{4}^{2}=8 \text {. }
$$

Conversely, when $a_{2} a_{3} a_{4} \neq 0$, and (9.6) and (9.8) hold, (9.7) satisfies (8.2) and $K=0$. Therefore we have

Proposition 9. 2. Let $K=0$ hold. If we assume $f=2 M^{-1}$ and $(\beta, \gamma$, $\delta \neq)$, the solution of (8.2) is given by (9.7), where a's are arbitrary nonvanishing constants satisfying (9.6) and (9.8). In this case, we have

$$
B=c_{2} M^{\left(2+a_{2}-a_{4}\right) / 3}, \quad C=c_{3} M^{\left(2+a_{3}-a_{2}\right) / 3}, \quad D=c_{4} M^{\left(2+a_{4}-a_{3}\right) / 3} .
$$

The actual method of obtaining $a$ 's is as follows: If we eliminate $a_{2}$ from (9.6) and (9. 8), we have

$$
a_{3}{ }^{2}+a_{4}{ }^{2}+a_{3} a_{4}=4 \text {. }
$$

Take $a_{3}$ and $a_{4}$ satisfying $a_{3} a_{4} \neq 0$ and (9.10), and determine $a_{2}$ from (9. 8). Then this set $\left(a_{2}, a_{3}, a_{4}\right)$ gives the solution, if $a_{2} \neq 0$. An example is given by 


$$
\begin{aligned}
& a_{2}=1, \quad a_{3}=(-1+\sqrt{13}) / 2, \quad a_{4}=-(1+\sqrt{13}) / 2 ; \\
& \beta=(7+\sqrt{13}) / 6 M, \quad \gamma=(1+\sqrt{13}) / 6 M, \quad \delta=(2-\sqrt{13}) / 3 M ; \\
& B=c_{2} M^{(7+\sqrt{13}) / 6}, \quad C=c_{3} M^{(1+\sqrt{13}) / 6}, \quad D=c_{4} M^{(2-\sqrt{13}) / 3} .
\end{aligned}
$$

Further we have

Proposition 9.3. $\boldsymbol{K}_{\dot{A}}^{B}$ of $V_{\mathrm{V}}$ stated in Proposition 9.2 has 3 double eigenvalues, and these values are not constants. (As a result, they are non-vanishing.)

The proof is easy if we use the fact that, for example, $\beta \delta=\gamma \delta$ is equivalent to $\delta=0$, i. e. $a_{3}-a_{4}=2$, which together with (9.10) gives $a_{3} a_{4}=0$.

$\left(i i_{4}\right)$ Next, we assume $f=2 M^{-1}$ and $(\beta=\gamma \neq \delta)$. Just as in $(i)$, we obtain from (8.2)

$$
\delta-\beta=a_{4} M^{-1} \text {. }
$$

Making use of (8.2) again, we find that $(\beta=\gamma, \delta)$ and $(B, C, D)$ must be one of the following two types:

$$
\begin{array}{ll}
\left(i i_{4 a}\right) \quad a_{4}=2, \quad \beta=\gamma=0, \quad \delta=2 M^{-1} ; \quad B=c_{2}, \quad C=c_{3}, \quad D=c_{4} M^{2} . \\
\left(i_{4 b}\right) \quad a_{4}=-2, \quad \beta=\gamma=(4 / 3) M^{-1}, \quad \delta=-(2 / 3) M^{-1} ; \\
B=c_{2} M^{4 / 3}, \quad C=c_{3} M^{4 / 3} \quad D=c_{4} M^{-2 / 3}
\end{array}
$$

$\left(i i_{4 a}\right)$ is nothing but the one given in $\left(\mathrm{III}_{4 a}\right)$ of Propositions 8.2 and 8.3 , and $\boldsymbol{K}_{A}^{\cdot B}$ has a sextuple eigenvalue $0 . \quad \boldsymbol{K}_{A}^{B}$ of $\left(i i_{4 b}\right)$ has a quadruple eigenvalue -(2/9) $M^{-2}$ and a double eigenvalue (4/9) $M^{-2}$. Both eigenvalues are functions of $x$, and are not constants.

$\left(i i_{2}\right)$ We now assume $f=2 M^{-1}$ and $(\beta \neq \gamma=\delta)$. (The case of $\left(i i_{3}\right)(\gamma \neq \beta$ $=\delta$ ) can be obtained from the present one by a simple change.) Just as in $\left(i i_{4}\right)$, we can obtain, by making use of $\beta-\gamma=a_{2} / M$, the following two kinds of solutions :

$$
\begin{array}{ll}
\left(i i_{2 a}\right) \quad a_{2}=2, \quad \gamma=\delta=0, \quad \beta=2 M^{-1} ; \quad B=c_{2} M^{2}, \quad C=c_{3}, \quad D=c_{4}, \\
\left(i i_{2 b}\right) \quad \begin{array}{l}
a_{2}=-2, \quad \beta=-(2 / 3) M^{-1}, \quad \gamma=\delta=(4 / 3) M^{-1} \\
B=c_{2} M^{-2 / 3}, \quad C=c_{3} M^{4 / 3}, \quad D=c_{4} M^{4 / 3} .
\end{array}
\end{array}
$$

$\left(i i_{2 a}\right)$ is the one in $\left(\mathrm{III}_{2 a}\right)$ of Propositions 8.2 and 8.3. Concerning the eigenvalues of $\boldsymbol{K}_{\dot{A}}^{\cdot B}$ of $\left(i i_{2 b}\right)$, we have the same results as in $\left(i i_{4 b}\right)$.

(iii) The case in which $f=2 M^{-1}$ and $\beta=\gamma=\delta$ hold. We have $\beta=\gamma$ $=\delta=(2 / 3) M^{-1}$. Since this $\beta$ does not satisfy $2 \beta^{\prime}+\beta^{2}=\beta^{2}$, i. e. $\beta^{\prime}=0$, we cannot have such a solution. 
Summarizing the results obtained above, we have

Proposition 9. 4. When $K=0$, the solutions of (8.2) are given by those stated in $\left(i i_{\rho a}\right)$ and $\left(i i_{\rho b}\right),(\rho=2,3,4)$, if we assume $f=2 M^{-1}$ and exclude the case of $(\beta, \gamma, \delta \neq)$. The $V_{\mathrm{v}}$ 's in $\left(i i_{\rho a}\right)$ are $S(B)$, while those in $\left(i i_{\rho b}\right)$ are not $S(B) . \quad \boldsymbol{K}_{A}^{\cdot}$ of $\left(i i_{p b}\right)$ has one quadruple eigenvalue $\nu$ and one double eigenvalue $\nu^{\prime}(=-2 \nu)$, where $\nu$ is a non-constant function.

We do not restate, with use of the members of c.s., the results obtained above. But it should be noted that the gradients of eigenvalues of $\boldsymbol{K}_{A}^{B}$ in Proposition 9.3 and those in $\left(i i_{\rho b}\right)$ of Proposition 9.4 are proportional to $u_{i}$. As is stated at the beginning of this section, these space-times are non-flat exact solutions of the Einstein equation (9.3).

Thus we have completed the study of the Einstein $V_{\nabla}$ satisfying $K=0$.

\section{$\S 10$. Some preparatory propositions, 5 .}

In this last section, we consider the case of the Einstein $V_{\mathrm{v}}$ satisfying $K \neq 0$, the last case remained. Then we have, corresponding to (9.3),

$$
K_{i j}=(K / 4) g_{i j} \text {, }
$$

where $K / 4=\nu=$ const. (cf. Proposition 8, 1). (10.1) is nothing but the Einstein field equation with a cosmological term. So, again the results of this section will be of some meanings from the physical point of view. The results obtained in $\S 8$ concerning $S(A)$ will be included in those of this section.

(B) We first assume $K<0$, and put $p=\sqrt{-3 K} / 2$. Thus $p$ is a nonvanishing constant. Then from (9.2), we have

$$
v=a e^{p x}+b e^{-p x},
$$

where $a$ and $b$ are arbitrary constants which do not satisfy $a=b=0$. Then we can obtain from (8.2)

$$
\begin{array}{ll}
\beta=v^{-1}\left\{a_{2}+(2 p / 3) w\right\}, \quad \gamma=v^{-1}\left\{a_{3}+(2 p / 3) w\right\}, \\
\delta=v^{-1}\left\{a_{4}+(2 p / 3) w\right\}, \quad w \equiv a e^{p x}-b e^{-p x}, \quad\left(v^{\prime}=p w, w^{\prime}=p v\right),
\end{array}
$$

where $a$ 's are arbitrary constants satisfying

$$
a_{2}+a_{3}+a_{4}=0 \text {. }
$$

Again substituting (10.3) into (8.2), we find that a necessary and sufficient condition that $\beta, \gamma, \delta$ given by (10.3) satisfy (8.2), is given by (10.4) and 
(10.5)

$$
a_{2} a_{3}+a_{3} a_{4}+a_{4} a_{2}=16 a b p^{2} / 3=-4 a b K .
$$

From (10.3), we have

$$
B=c_{2} v^{2 / 3} \exp \left(a_{2} F\right), \quad C=c_{3} v^{2 / 3} \exp \left(a_{3} F\right), \quad D=c_{4} v^{2 / 3} \exp \left(a_{4} F\right),
$$

where $F=\int v^{-1} d x=\int\left(a e^{p x}+b e^{-p x}\right)^{-1} d x$. Therefore we have

Proposition 10.1. When $K<0$, the general forms of $(\beta, \gamma, \delta)$ and $(B, C, D)$ satisfying (8.2) are given by (10.3) and (10.6) respectively, where a's are arbitrary constants satisfying (10.4) and (10.5).

Especially when $b=0$ (or $a=0)$ and $\beta=\gamma=\delta$, we have $a_{2}=a_{3}=a_{4}=0$ and

$$
\beta=\gamma=\delta=2 p / 3 ; \quad B=c_{2} G, C=c_{3} G, D=c_{4} G, \quad\left(G=e^{2 p x / 3}\right),
$$

where $c$ 's are new arbitrary positive constants. (10.7) is nothing but those given by (8.6) in which $p$ is replaced by $2 p / 3$. In connection with this, we have

Proposition 10.2. When $K<0, V_{\mathrm{v}}$ must be $S(A)$, if $\beta=\gamma=\delta$ holds.

Proof. From (10.3) and (10.4), we have $a_{2}=a_{3}=a_{4}=0$, and from (10.5), $a b=0$. Then it is evident that the $V_{\mathrm{v}}$ is $S(A)$ (cf. Proposition 8. 4). Q.E.D.

It is easy to show that we have solutions $\left(a_{2}, a_{3}, a_{4}\right)$ of $(10.4)$ and (10.5) giving various types: $(i)(\beta, \gamma, \delta \neq),\left(i i_{2}\right)(\beta \neq \gamma=\delta), \cdots$. Examples are

(i) $\left\{a_{2}=a_{3} / 2=-a_{4} / 3=(4 / \sqrt{21}) p \sqrt{-a b}\right\},\left(i i_{2}\right)\left\{-a_{2} / 2=a_{3}=a_{4}=(4 / 3) p \sqrt{-a b}\right\}, \cdots$.

Proposition 10.3. A necessary and sufficient condition that we have a real solution of (10.4) and (10.5) is $a b \leqq 0$.

Proof. By virtue of (10.4), we have $a_{2} a_{3}+a_{3} a_{4}+a_{4} a_{2}=-\left(a_{2}{ }^{2}+a_{3}{ }^{2}+a_{4}{ }^{2}\right) / 2$ and hence from (10.5), the proposition follows. Q.E.D.

Thus we can say that the space-time under consideration cannot be $V_{\mathbf{V}}$ in our sense when $a b>0$. Evidently $\boldsymbol{K}_{A}^{B}$ has a sextuple eigenvalue for $S(A)$, a quadruple and a double eigenvalues when two of $\beta, \gamma, \delta$ coincide, and three double eigenvalues when $(\beta, \gamma, \delta \neq)$. Except the case of $S(A)$, the eigenvalues are non-constant functions of $x$.

(C) Lastly, we consider the case $K>0$, and put $\sqrt{3 K} / 2=p$. Then, corresponding to (10.2), we have from (9.2)

$$
v=a \sin p x+b \cos p x,
$$

where $a$ and $b$ are arbitrary constants, at least one of which is non-vanishing. The equations corresponding to (10.3), (10.4) and (10.5) are respectively 


$$
\begin{array}{ll}
\beta=v^{-1}\left\{a_{2}+(2 p / 3) w\right\}, & \gamma=v^{-1}\left\{a_{3}+(2 p / 3) w\right\}, \\
\delta=v^{-1}\left\{a_{4}+(2 \mathrm{p} / 3) w\right\} ; & w=a \cos p x-b \sin p x, \quad\left(v^{\prime}=p w, w^{\prime}=-p v\right),
\end{array}
$$

$$
a_{2}+a_{3}+a_{4}=0 \text {, }
$$

$$
a_{2} a_{3}+a_{3} a_{4}+a_{4} a_{2}=-(4 / 3) p^{2}\left(a^{2}+b^{2}\right)=-\left(a^{2}+b^{2}\right) K .
$$

The actual forms of $B, C$ and $D$ are given by (10.6) with $F=\int v^{-1} d x=$ $\int(a \sin p x+b \cos p x)^{-1} d x$.

If we put $\beta=\gamma=\delta$, we have from (10.10) and (10.11), $a_{2}=a_{3}=a_{4}=a$ $=b=0$, which cannot be the case. Therefore the $V_{\mathrm{V}}$ cannot be $S(A)$, in conformity with the result in Proposition 8.4. Similarly to the preceding case, we can show the actual examples of the solutions of (10.10) and (10.11) of type $(i)$ and $\left(i i_{\rho}\right)$. (As is stated in the above, we cannot have the type (iii).) The eigenvalues of $\boldsymbol{K}_{A}^{B}$ are of type $\{1$ double and 1 quadruple $\}$ or $\{3$ double $\}$-values and these values are not constants.

Thus in $\S 8, \S 9$ and the present section, we have completed the preparatory investigations concerning the Einstein $V$ 's. When $\boldsymbol{K}_{A}^{B}$ has a sextuple eigenvalue, the $V$ is $S(B)$ or $S(A)$. The value is 0 for $S(B)$ and a non-vanishing constant for $S(A)$. If we exclude such cases, the remaining $V_{\mathrm{v}}$ 's are of type $\{1$ double and 1 quadruple $\}$ - or $\{3$ double $\}$-eigenvalues, and further the values are non-constant functions of $x$ in all cases. This result will be used in considering the freedom of c.s. in these $V_{\mathrm{v}}$ 's.

$$
\begin{array}{cc}
\text { Research Institute for Theoretical Physics } & \\
\text { Hiroshima University } & \text { and Fukui University } \\
\text { Takehara-shi, Hiroshima-ken } & \text { Fukui-shi }
\end{array}
$$

\section{References}

[1] H. TAkeno and S. Kitamura: On some special kind of space-times, I, Tensor, N.S. (In the press).

[2] H. TAKENO: The theory of spherically symmetric space-times (Revised edition), Sci. Rep. Res. Inst. Theor. Phys., Hiroshima Univ., No. 5 (1966).

[3] A. G. WALkER: On Ruse's spaces of recurrent curvature, Proc. London Math. Soc., 52 (1950), 36-64.

[4] L. P. Eisenhart: Riemannian geometry, Princeton Univ. Press, (1949). 